\title{
MULTIPLE INTEGRALS OF LIPSCHITZ FUNCTIONS IN THE CALCULUS OF VARIATIONS
}

\author{
FRANK H. CLARKE
}

\begin{abstract}
We consider a multiple integral problem in the calculus of variations in which the integrand is locally Lipschitz but not differentiable, and in which minimization takes place over a Sobolev space. Using a minimax theorem, we derive an analogue of the classical Euler condition for optimality, couched in terms of "generalized gradients". We proceed to indicate how these results may be applied to deduce existence and smoothness properties of solutions to certain Poisson equations.
\end{abstract}

1. Introduction Let a function $f: R \times R^{\nu} \rightarrow R$ and a domain $G$ in $R^{\nu}$ be given. We shall be considering the following multiple integral problem in the calculus of variations: to minimize

$$
\int_{G} f(z(x), \nabla z(x)) d x
$$

over a certain class of functions $z$ defined on $G$. In dealing with this problem we have adopted the notation and terminology of Morrey [4] whenever possible. Our concern will be to obtain necessary conditions for optimality in the absence of differentiability hypotheses on the function $f$. Several examples of problems involving nondifferentiable integrands are given in Chapter 4 of [3], in which the methods of convex analysis are applied. However, we make no convexity assumptions in this article.

The medium in which these new necessary conditions are expressed is the "generalized gradient" introduced in [1]. We now define this for a locally Lipschitz function $g: R^{k} \rightarrow R$ (i.e. $g$ satisfies a uniform Lipschitz condition on every bounded subset of $R^{k}$ ). Given a point $x$, the generalized gradient of $g$ at $x$, denoted $\partial g(x)$, is the set $\operatorname{co}\left\{\zeta: \zeta=\lim _{i \rightarrow \infty} \nabla g\left(x_{i}\right), \lim _{i \rightarrow \infty} x_{i}=x\right\}$. That is, we consider all sequences $\left\{x_{i}\right\}$ converging to $x$ such that $\nabla g\left(x_{i}\right)$ exists for each $i$ and such that the indicated limit $\zeta$ exists. The convex hull of all the points $\zeta$ so obtained is $\partial g(x)$. We obtain in this way a nonempty compact convex set [1]. Furthermore, $\partial g(x)$ reduces to $\{\nabla g(x)\}$ when $g$ is $C^{1}$, and when $g$ is convex $\partial g(x)$ is the subdifferential of $g$ at $x$.

Let $H$ be a subset of the Sobolev space $H_{1}^{1}(G)$ (see [4]) with the property of

Received by the editors June 28, 1976.

AMS (MOS) subject classifications (1970). Primary 49B20, 49B25, 35A15.

Key words and phrases. Multiple integrals, nondifferentiable functions, Euler-Lagrange equation, generalized gradients, Poisson's equation.

${ }^{1}$ Sponsored by the United States Army under Contract No. DAAG29-75-C-0024, while the author was visiting the Mathematics Research Center, University of Wisconsin, Madison. 
being closed under addition of elements of $C^{\infty}(G)$; i.e. $h+\phi$ belongs to $H$ for every $h$ in $H$ and $\phi$ in $C^{\infty}(G)$. We suppose that the element $z$ of $H$ furnishes a weak local minimum for (1.1) over all functions in $H$ assuming the same values on the boundary of $G$ (see [4, Definition 3.3.1]). Our main result will be proven under either of the two following hypotheses:

$\left(\mathrm{H}_{1}\right) f(s, p)>1$ for all $(s, p)$ in $R \times R^{\nu}$, and for some constant $K$, for all $(s, p)$ and $(\alpha, \beta)$ in $R \times R^{v}$, we have

$$
f(s+\alpha, p+\beta)<f(s, p) \exp \{K|(\alpha, \beta)|\}+K[1+|(s, p)|]|(\alpha, \beta)| .
$$

$\left(\mathrm{H}_{2}\right) z$ and $\nabla z$ belong to $L^{\infty}(G)$, and $f$ is locally Lipschitz.

THEOREM. If $z$ is optimal as described above, and if either $\left(\mathrm{H}_{1}\right)$ or $\left(\mathrm{H}_{2}\right)$ holds, there exists a function $\zeta: G \rightarrow R^{\prime}$ such that each component of $\zeta$ belongs to $L^{1}(G), \operatorname{div} \zeta$ belongs to $L^{1}(G)$, and

$$
(\operatorname{div} \zeta(x), \zeta(x)) \in \partial f(z(x), \nabla z(x)) \text { a.e. in } G .
$$

Remark 1. If $f$ is differentiable, $\left(H_{1}\right)$ is closely related to the growth condition

$$
\left|f^{\prime}(s, p)\right| \leqslant K f(s, p)+K[1+|(s, p)|] .
$$

Since $\left(\mathrm{H}_{1}\right)$ implies that $f$ is locally Lipschitz, the generalized gradient in (1.2) is defined whether $\left(\mathrm{H}_{1}\right)$ or $\left(\mathrm{H}_{2}\right)$ holds.

REMARK 2. When $f$ is $C^{1},(1.2)$ yields the Euler-Lagrange equation

$$
\operatorname{div}\left\{\nabla_{p} f(z, \nabla z)\right\}=\nabla_{z} f(z, \nabla z),
$$

since the generalized gradient then coincides with the gradient. We thus obtain the classical necessary condition under reduced regularity hypotheses. The proof of the theorem (which is given in \$2) uses an abstract minimax theorem. The application of a minimax theorem in such a context appears new.

\section{Proof of the theorem.}

LEMMA 1. There exists a function $\bar{K}(x)$ in $L^{1}(G)$ with the following property: for almost every $x$ in $G$, for every pair of points $\left(s_{1}, p_{1}\right)$ and $\left(s_{2}, p_{2}\right)$ in $R \times R^{\text {v }}$ satisfying $\left|\left(s_{i}-z(x), p_{i}-\nabla z(x)\right)\right|<1(i=1,2)$, we have

$$
\left|f\left(s_{1}, p_{1}\right)-f\left(s_{2}, p_{2}\right)\right| \leqslant \bar{K}(x)\left|\left(s_{1}-s_{2}, p_{1}-p_{2}\right)\right| .
$$

Proof. When $\left(\mathrm{H}_{2}\right)$ holds, the lemma is trivially true, so let us posit $\left(\mathrm{H}_{1}\right)$. Then $f\left(s_{1}, p_{1}\right)-f\left(s_{2}, p_{2}\right)$ is bounded above by

$$
f\left(s_{2}, p_{2}\right)[\exp \{K \Delta\}-1]+K\left[1+\left|\left(s_{1}, p_{1}\right)\right|\right] \Delta
$$

where $\Delta$ is $\left|\left(s_{1}-p_{1}, s_{2}-p_{2}\right)\right|$. In turn (using $\left(\mathrm{H}_{1}\right)$ again) (2.2) is no greater than 


$$
\begin{aligned}
\{f(z, \nabla z) \exp \{K\}+K[1+|(z, \nabla z)|]\}[\exp \{K \Delta\}-1] & \\
& +K[2+|(z, \nabla z)|] \Delta .
\end{aligned}
$$

Since $\exp \{K \Delta\}-1$ is no larger than $K \exp \{2 K\} \Delta$ (by elementary calculus), this last expression is bounded above by

$$
\{\alpha f(z, \nabla z)+\beta|(z, \nabla z)|+\gamma\} \Delta
$$

for certain constants $\alpha, \beta$ and $\gamma$. We can repeat this argument with the roles of $\left(s_{1}, p_{1}\right)$ and $\left(s_{2}, p_{2}\right)$ reversed, and the required conclusion then follows by letting $\bar{K}(x)$ be the coefficient of $\Delta$ in (2.3). (Note that $f(z, \nabla z)$ is in $L^{1}(G)$ by hypothesis.) Q.E.D.

Let us define $A$ to be the set of all (Lebesgue) measurable functions $(\varphi(x), \zeta(x))$ defined on $G$ such that $(\varphi(x), \zeta(x)) \in \partial f(z(x), \nabla z(x))$ a.e. in $G$. It follows from Lemma 1 that the set $\partial f(z(x), \nabla z(x))$ is bounded by $\bar{K}(x)$, so that $A$ is a subset of $L^{1}(G)^{\nu+1}$, which we endow with its weak topology.

Lemma 2. $A$ is a nonempty convex weakly compact subset of $L^{1}(G)^{\nu+1}$.

Proof. Since $\partial f$ is a convex set, $A$ is evidently convex. If a subsequence in $A$ converges strongly in $L^{1}(G)^{\nu+1}$, the limit must lie in $A$ (since a subsequence converges a.e. and $\partial f$ is closed). This implies that $A$ is closed in the strong topology. This (together with the convexity of $A$ ) implies that $A$ is weakly closed. It follows from the Dunford-Pettis criterion that $A$ is weakly precompact, so we deduce weak compactness. That $A$ is nonempty follows from the fact that the multifunction $x \rightarrow \partial f(z(x), \nabla z(x))$ is measurable (as a consequence of [1, Proposition 1.4]) and hence admits a measurable selection [5]. Q.E.D.

We now define $B$ to be the (convex) set of all $C^{\infty}$ functions having compact support in $G$, and we define $L: A \times B \rightarrow R$ as follows (for $a=$ $(\varphi, \zeta))$ :

$$
L(a, b)=\int_{G}\{\varphi(x) b(x)+\langle\zeta(x), \nabla b(x)\rangle\} d x .
$$

Note that $L$ is well defined since $\varphi$ and $\zeta$ are integrable. It is evident that for each $b$ in $B, L(\cdot, b)$ is continuous on $A$ (in the weak topology).

LEMMA 3. $\inf _{b \in B} \sup _{a \in A} L(a, b)=0$.

Proof. Since $L(\cdot, 0)$ is identically 0 , it suffices to prove that for any given $b$ in $B$, the following quantity is nonnegative:

$$
\sup _{a \in A} L(a, b) \text {. }
$$

We proceed to prove this. From measurable selections it follows that (2.4) is equal to 


$$
\int_{G} \sup \{\varphi b(x)+\langle\zeta, \nabla b(x)\rangle:(\varphi, \zeta) \in \partial f(z(x), \nabla z(x))\} d x,
$$

and this in turn majorizes (by [1, Proposition 1.4])

$$
\int_{G} \limsup _{\lambda \downarrow 0}[f(z+\lambda b, \nabla z+\lambda \nabla b)-f(z, \nabla z)] / \lambda d x .
$$

For each $\lambda$ sufficiently small, the integrand in (2.5) is bounded pointwise by $\bar{K}(x)$ (in view of Lemma 1). Consequently we may apply Fatou's lemma to deduce that $(2.5)$ is no less than

$$
\underset{\lambda \downarrow 0}{\lim \sup } \int_{G}[f(z+\lambda b, \nabla z+\lambda \nabla b)-f(z, \nabla z)] / \lambda d x
$$

But this expression is certainly nonnegative, since (for $\lambda$ small) $z+\lambda b$ belongs to $H$, satisfies the boundary conditions, and lies within the weak neighborhood relative to which $z$ minimizes (1.1). Q.E.D.

We shall now apply a "lop-sided" version of the Sion-Ky Fan minimax theorem, which we state here for convenience. A proof of this result may be found in [2].

TheOREM Let $A$ and $B$ be nonempty convex subsets of two locally convex topological vector spaces, and let $A$ be compact. Suppose that $L: A \times B \rightarrow R$ is such that for each $a \in A, L(a, \cdot)$ is convex, and for each $b \in B, L(\cdot, b)$ is upper semicontinuous and concave. Then, if the quantity

$$
\beta=\inf _{b \in B} \sup _{a \in A} L(a, b)
$$

is finite, we have $\beta=\sup _{a \in A} \inf _{b \in B} L(a, b)$, and there exists an element $a \in A$ such that $\inf _{b \in B} L(a, b)=\beta$.

On applying the above theorem (with $\beta=0$ ) to our present situation, we deduce that there exists an element $(\varphi, \zeta)$ of $A$ such that

$$
\int_{G}\{\varphi b+\langle\zeta, \nabla b\rangle\} d x \geqslant 0
$$

for all $b$ in $B$. Since $-B=B,(2.6)$ must in fact hold with equality for all $b$. By definition, $\varphi=\operatorname{div} \zeta$. Q.E.D.

REMARK. Only slight modifications are required to handle the case in which $f$ has an explicit dependence on $x$. It would suffice to have $\left(\mathrm{H}_{1}\right)$ or $\left(\mathrm{H}_{2}\right)$ hold uniformly in $x$, and to suppose that for each $(s, p)$ the function $x \rightarrow f(x, s, p)$ is measurable.

3. An application of the theorem. It is well known that existence and regularity theorems for partial differential equations can be obtained through variational methods. We illustrate in this section how the above theorem can be used in much the same way, but with the added possibility of introducing nondifferentiable functions.

Let $E$ be a given multifunction mapping $R$ to the subsets of $R$, and consider the "Poisson inclusion" 


$$
\Delta z(x) \in E(z(x)) \text { a.e. in } G,
$$

where $\Delta$ is the Laplacian. The connection to the theorem is the following: If $g: R \rightarrow R$ is a Lipschitz function such that $\partial g(s)=E(s)$ for $s$ in $R$, then relation (1.2) is equivalent to (3.1) if $f$ is defined by $f(s, p)=g(s)+|p|^{2} / 2$.

ExAmple 1. Let $G$ be bounded. Suppose we seek $z$ in $H_{1}^{1}(G)$ assuming given boundary values and satisfying

$$
\begin{aligned}
|\Delta z| & \leqslant 1 & & \text { in } G, \\
\Delta z & =1 & & \text { if } z>0 \\
& =-1 & & \text { if } z<0 .
\end{aligned}
$$

(That is, $E(s)=\{1\}$ if $s>0,\{-1\}$ if $s<0$ and $[-1,1]$ if $s=0$.) We consider the problem of minimizing $\int_{G}\left\{|z(x)|+|\nabla z(x)|^{2} / 2\right\} d x$ over $H_{1}^{1}(G)$ subject to the given boundary conditions. By [4, Theorem 1.9.1] there exists a (unique) solution $z$ to this problem. We now apply the theorem, and we see that $z$ satisfies the required conditions. For other methods of treating problems similar to this, we refer the reader to [6].

EXAMPLE 2. Let $g$ be locally Lipschitz, let $\nu \leqslant 3$, and let $z$ minimize $\int_{G}\left\{g(z(x))+|\nabla z(x)|^{2} / 2\right\} d x$ over $H_{1}^{1}(G)$, subject to given boundary conditions. Then if $z$ is bounded, $z$ is continuous.

To prove this, we begin by noting that $g(s)+|p|^{2} / 2$ satisfies $\left(\mathrm{H}_{1}\right)$ in any weak neighborhood of $z$, which is all that is needed to apply the theorem. We find as before that $\zeta=\nabla z$, so that $\operatorname{div} \zeta=\Delta z$ belongs to $\partial g(z(x))$ a.e. Since $z$ is bounded, this implies that $\Delta z$ is bounded in $G$. But then [6, Theorem 27.2] we may assert that $z$ belongs to $H_{2}^{2}(G)$ (we assume here some regularity of $\partial G$ and the boundary conditions). That $z$ is continuous now follows from Sobolev's lemma [6, Theorem 24.2]. Q.E.D.

ACKNOwledgement. I wish to thank Monsieur J. M. Lasry for several helpful discussions.

\section{REFERENCES}

1. F. H. Clarke, Generalized gradients and applications, Trans. Amer. Math. Soc. 205 (1975), 247-262. MR 51 \#3373.

2. I. Ekeland, Théorie des jeux, Presses Univ. France, Paris, 1975.

3. I. Ekeland and R. Temam, Analyse convexe et calcul des variations, Dunod; Gauthier-Villars, Paris, 1974.

4. C. B. Morrey, Jr., Multiple integrals in the calculus of variations, Springer-Verlag, Berlin and New York, 1966. MR 34 \#2380.

5. R. T. Rockafellar, Measurable dependence of convex sets and functions on parameters, J. Math. Anal. Appl. 28 (1969), 4-25. MR 40 \#288.

6. F. Treves, Basic linear partial differential equations, Academic Press, New York, 1975.

Department of Mathematics, University of British Columbia, Vancouver, B. C., Canada V6T IW5 